\title{
Maxime Decout, Éloge du mauvais lecteur
}

Paris, Minuit, coll. « Paradoxe », 2021

\section{Estelle Mouton-Rovira}

\section{(2) OpenEdition}

\section{Journals}

Édition électronique

URL : https://journals.openedition.org/recherchestravaux/3768

DOI : $10.4000 /$ recherchestravaux.3768

ISSN : 1969-6434

Éditeur

UGA Éditions/Université Grenoble Alpes

Édition imprimée

ISBN : 978-2-37747-301-4

ISSN : 0151-1874

Référence électronique

Estelle Mouton-Rovira, « Maxime Decout, Éloge du mauvais lecteur », Recherches \& Travaux [En ligne], 98 | 2021, mis en ligne le 28 juin 2021, consulté le 09 août 2021. URL : http://journals.openedition.org/ recherchestravaux/3768; DOI : https://doi.org/10.4000/recherchestravaux.3768

Ce document a été généré automatiquement le 9 août 2021.

(c) Recherches \& Travaux 


\section{Maxime Decout, Éloge du mauvais lecteur}

Paris, Minuit, coll. « Paradoxe », 2021

Estelle Mouton-Rovira

\section{RÉFÉRENCE}

Maxime Decout, Éloge du mauvais lecteur, Paris, Minuit, coll. « Paradoxe », 2021

1 Ce bel essai de Maxime Decout, publié chez Minuit au printemps 2021, emprunte la forme de l'éloge paradoxal pour chercher à saisir les traits d'un intrus, ou plutôt d'une figure habituellement tenue dans l'ombre par la théorie et la critique littéraires, celle $\mathrm{du}$ « mauvais lecteur».

2 Une telle réhabilitation de modes de lecture habituellement tenus pour répréhensibles est d'emblée historicisée par un premier chapitre («Mort et renaissance du mauvais lecteur ») qui rappelle l'histoire - ancienne - de la dévalorisation moderne de la lecture d'adhésion ou immersive, accusée de corrompre la morale des lecteurs et notamment des lectrices, et qui retrace l'émergence de la figure du bon lecteur, forgée par les théories de la lecture au $\mathrm{xx}^{\mathrm{e}}$ siècle. Maxime Decout souligne alors une tension entre l'horizon d'une lecture critique, représentée par un lecteur-type, par définition désincarné, et la résurgence d'une lecture identificatoire, parfois associée à l'enfance et légitimée par le récit qu'en font les écrivains (par exemple Proust, ou Sartre).

3 Si cette opposition entre une lecture commentatrice, analytique, et une lecture immersive a fait l'objet de tentatives de conciliation - l'auteur cite notamment les Fragments d'un discours amoureux de Barthes (1977) -, elle n'en reste pas moins irrésolue. Maxime Decout propose alors de décliner les nuances de cette lecture qui s'écarte des conventions théoriques, afin d'en essayer les postures et d'en éprouver les variations, autour d'un principe commun, celui du refus de l'idéal d'un déchiffrement normatif. Le deuxième chapitre («Splendeurs et misères de l'interprétation ») fait ainsi le portrait contrasté du lecteur «trop savant » (p.40), dont les représentations littéraires, à partir 
du XIX $x^{e}$ siècle, soulignent la richesse, et dont les traits se confondent parfois avec ceux d'un lecteur happé par son objet et victime d'une immersion intense. Du Motif dans le tapis de Henry James (1896) au Démolir Nisard d'Éric Chevillard (2006), le lecteur visé est alors celui qui, par obsession du commentaire, se plonge dans son objet d'étude jusqu'à la confusion; il est aussi celui qui, pugnace, se livre au déchiffrement contrefactuel afin d'inverser, par la lecture, le régime habituel de la preuve, comme le personnage de Pierre Senges, Antonio de Guevara, qui démontre l'inexistence de l'Amérique dans La Réfutation majeure (2004).

4 C'est alors la littérature qui, radicalisant la « surinterprétation » (Eco), court-circuite la théorie et se nourrit de la figure du commentateur. Ces mises en fiction de la lecture représentent en fin de compte une herméneutique déréglée qui confine à l'obsession, comme le montre le troisième chapitre («La parole au mauvais lecteur»). Les narrateurs de Cinéma (1999) et de La Disparition de Jim Sullivan (2013) chez Tanguy Viel, deviennent ainsi des lecteurs fétichistes, quand ceux de Feu Pâle, de Nabokov (1962), ou de L'euvre posthume de Thomas Pilaster, d'Éric Chevillard (1999), renversent le geste du commentaire savant et, parce qu'ils semblent se venger, par le commentaire, de l'auteur qu'ils annotent, mettent en évidence la virulence potentielle d'une pratique presque pulsionnelle de la lecture. Autrement dit, les textes que recense l'essai mettent en scène des pratiques de lecture atypiques ou hybrides - s'il s'agit bien souvent aussi de figures d'auteurs, narrateurs, commentateurs, éditeurs ou critiques, tous révèlent, sous le vernis savant, les signes d'une lecture plus personnelle, affective voire obsessionnelle.

Un tel parcours permet alors à Maxime Decout de souligner que la mauvaise lecture, parce qu'elle autorise un usage irrationnel du texte, comprend une part fantasmatique et reflète inévitablement la singularité de chaque lecteur. En conséquence, les méthodes de l'interprétation s'en trouvent réévaluées et relativisées dans leur prétention à l'objectivité, en vertu d'une réactualisation du texte par chaque lecture, qui produit alors un "texte fantôme", inventé par le lecteur. Enfin, le quatrième chapitre ("Les pratiques du mauvais lecteur ») invite à libérer la lecture des codes qui la restreignent, au profit de deux types de conduite, "la lecture buissonnière » et la «lecture interventionniste» (p. 105). Lire dans le désordre, lire « en puzzle», comme Perec l'espérait pour La Vie mode d'emploi, lire par fragments serait alors autant de parcours possibles dans les livres - si tant est que la lecture n'y soit pas déjà délinéarisée, comme chez Cortázar ou Calvino. C'est enfin l'idéal d'une lecture-écriture que l'essai met en valeur, jusqu'aux exemples empruntés à Philip Roth, Enrique VilaMatas ou Stephen King, dont les personnages rêvent d'intervenir directement dans les récits qu'ils lisent et témoignent de la persistance de l'idéal d'une littérature agissante (p. 142).

6 La figure du mauvais lecteur est donc, sous la plume de Maxime Decout, réinvestie à la fois comme motif productif pour le récit au $\mathrm{xx}^{\mathrm{e}}$ et au $\mathrm{xxI}^{\mathrm{e}}$ siècles, et comme reflet possible des traversées que chacun peut faire d'un livre, hors des conventions de la lecture critique. Le plaisir de la lecture s'en trouve décentré, à la manière d'une chasse au trésor dont on préfèrerait le parcours au résultat, l'exploration à la découverte. À ce titre, Maxime Decout rappelle que le roman policier postule un «Lecteur Modèle » qui soit un « mauvais lecteur » (p. 28), pour que la mécanique narrative fonctionne : c'est ce même plaisir du renversement qu'il semble poursuivre à travers les textes convoqués, qui composent en raccourci une bibliothèque enthousiasmante. Cette quête du mauvais 
lecteur, parce qu'elle congédie l'objectivité illusoire d'une interprétation individuelle, se noue à une réflexion sur la vérité littéraire : qu'il s'agisse de l'établir ou de la mettre en doute, la lecture n'y apporte point d'autre réponse que celle de sa mise en mouvement. On retrouve là un fil théorique commun aux précédents essais de Maxime Decout (En toute mauvaise foi. Sur un paradoxe littéraire, 2015; Qui a peur de l'imitation ?, 2017 ; Pouvoirs de l'imposture, 2018), qui explorent en somme la manière dont la fiction déjoue et déplace toute forme de certitude.

7 Les lecteurs que convoque l'essayiste ne sont d'ailleurs pas tant lecteurs qu'apprentis herméneutes, exégètes facétieux, ou commentateurs zélés: ce qui frappe, dans ce corpus, c'est la récurrence des figures de l'excès, des personnages de Bouvard et Pécuchet à ceux des nouvelles de Borges: leur démesure interprétative, si elle met en jeu les figures de l'amateur ou de l'autodidacte, les renverse au profit d'un mouvement illimité du commentaire, qui déstabilise toute entreprise de connaissance - dans des registres différents, 53 jours de Perec (1989) ou les Fragments de Lichtenberg de Pierre Senges (2008) le montrent bien. Il faut alors souligner le tact du geste critique, qui évite soigneusement d'en faire une analyse ironique ou d'en souligner le potentiel comique, ce qui aurait reconduit implicitement une norme axiologique par rapport à laquelle évaluer une transgression ou une subversion. Maxime Decout propose plutôt de voir dans ce personnel romanesque une gamme de conduites auxquelles l'essai, rédigé à la deuxième personne, suggère d'emboîter le pas.

La défense de l'interprétation fabulatrice, qui semble rassembler la lecture «bricoleuse» (p.130) et les formes de lecture créatrice, semble pourtant ramener la réflexion du côté d'une pratique lettrée des textes et de leurs prolongements possibles. Si l'image des textes fantômes, empruntée à Michel Charles, reste extrêmement stimulante, il semble pourtant que cet Éloge du mauvais lecteur défende, en creux, une appropriation créatrice des figures littéraires de la lecture. Si cette vision parvient à défaire habilement les soubassements axiologiques des pratiques de lecture savantes ou non, trop longtemps opposées, l'idéal d'une lecture-écriture semble se maintenir. À tout le moins, la valorisation d'une critique créatrice, elle-même tributaire d'une longue histoire, se prolonge en partie à travers ce "mauvais lecteur ", dont les réflexes et les compétences - l'indocilité, le soupçon, la ruse, mais aussi la faculté de se réinventer - sont aussi celles d'un fin lettré.

Enfin, si cet Éloge emporte l'adhésion, ce n'est pas tant parce qu'il convoque les figures plaisantes de lecteurs-resquilleurs, qui donnent d'ailleurs tout leur sel aux pages qu'ils peuplent, mais aussi parce qu'en rappelant la part fantasmatique et désirante de la lecture, il réaffirme la grande liberté des lecteurs devant les pages des livres, et s'inscrit fermement contre « l'imposition des significations par le texte ou la culture» (p. 145), en tenant à distance l'idée d'une loi du texte qui contraindrait l'interprétation à la restitution de visions du monde normatives. 


\section{AUTEURS}

\section{ESTELLE MOUTON-ROVIRA}

Université Bordeaux-Montaigne 